\title{
Photophysical performance of radio frequency sputtered Pt/n-PSi/ZnO NCs/Pt photovoltaic photodetectors
}

\begin{abstract}
The effect of the annealing temperature on the photoelectrical properties of the nanoporous silicon/zinc oxide nanocrystallites-based ( $\mathrm{Pt} / \mathrm{n}-\mathrm{PSi} / \mathrm{ZnO} \mathrm{NCs} / \mathrm{Pt})$ photodetector was investigated. Different morphologies of $3 \mathrm{D} \mathrm{ZnO}$ were synthesized onto the n-PSi substrates via radio frequency (RF) sputtering in the absence of a catalyst. The synthesis of $\mathrm{ZnO}$ NCs was controlled by varying the growth temperature between $600-700{ }^{\circ} \mathrm{C}$ and $800-900{ }^{\circ} \mathrm{C}$. The effect of the synthesis temperature on the structural, morphological, and optical properties of the n$\mathrm{PSi} / \mathrm{ZnO} \mathrm{NCs}$ was systematically studied using field emission scanning electron microscopy (FESEM), X-ray diffraction (XRD), atomic force microscopy (AFM), and photoluminescence spectroscopy (PL) techniques. The roughness was found to be dependent on the anodization current density. The optimal n-PSi/ZnO NCs-based metal-semiconductor-metal UV detector (MSM) was fabricated at $700{ }^{\circ} \mathrm{C}$. The fabricated device showed a high sensitivity of 1007.14 , an internal photoconductive gain of 11.07, and a responsivity of $5.99 \mathrm{~A} / \mathrm{W}$ with a low dark current when illuminated with $380 \mathrm{~nm}$ light $(1.55 \mathrm{~mW} / \mathrm{cm} 2)$ at $+5 \mathrm{~V}$ bias voltage. In addition, the response and recovery times were determined to be 0.34 and $0.22 \mathrm{~s}$, respectively. This approach offers a cost-effective substrate and simple synthesis method to improve the growth of the n-PSi/ZnO NCs and demonstrates the successful fabrication of nanoscale photodetectors with potential application in nano-optics devices.
\end{abstract}

Keyword: RF sputtering; $\mathrm{ZnO}$ nanocrystallites; Porous silicon; Annealing temperature; Photodetector 